\title{
Sentinel Lymph Node Mapping VS Systematic Lymphadenectomy for Endometrial Cancer: Surgical Morbidity and Lymphatic Complications.
}

\author{
Guilherme S. Accorsi, M.D; Luiza L. Paiva M.D; Ronald Schmidt M.D; Marcelo Vieira, \\ MSc; Ricardo Reis, PhD; Carlos Andrade, MSc. \\ Department Gynecologic Oncology of Barretos Cancer Hospital, Brazil.
}

INTRODUCTION: Today there is a lack of evidence reporting the incidence of complications from sentinel lymph node (SLN) in the management of endometrial cancer (EC). Our objective is to define complication rates of SLN biopsy in EC patients.

METHODS: We retrospectively analyzed all patients with endometrial cancer surgically treated at Barretos Cancer Hospital between April 2013 and March 2018. We evaluate intraoperative complication and 30-day complications separating the patients in four groups: Hysterectomy $(\mathrm{HT})$, hysterectomy plus pelvic lymphadenectomy, with or without para-aortic (HT+LND), hysterectomy plus sentinel biopsy $(H T+S L N)$ and hysterectomy plus lymphadenectomy and sentinel biopsy (HT+ $S L N+L N D$ ). Data were analyzed between the groups, and relationship of variables was verified using Multiple Logistic Regression.

RESULTS: Compared to HT group, HT+SLN did not show any increased risk of complications, intra-operative injury ( 0 vs 1 ; $p=1.0$ ) and 30-day complications ( 8 vs $7 ; p=0.782$ ). Surgical time was approximately 20 minutes longer $(p=0.016)$. The $\mathrm{HT}+\mathrm{LND}$ had an increased surgical time $(370 \mathrm{~min}, p<0.001)$, intraoperative blood-loss $(100 \mathrm{cc}, p<0.001)$, higher incidence of injury during surgery $(7 \%, p=0.005)$ and any 30 -day complication $(38.2 \%, p=0.002)$. Performing LND was associated with a greater risk of 30-day complications (HR 14.25; 95\% Cl 1.8519.63), intra-operative injury (HR 3.11; $95 \% \mathrm{Cl} 1.62-5.98)$, and lower limb lymphedema (LLL) (HR 8.14; 95\% Cl 1.01-65.27).
CONCLUSION: SLN mapping does not increase morbidity in surgical treatment of endometrial cancer patients. Our findings support the use of SLN algorithm, mainly in low risk endometrial cancer.

\begin{tabular}{|c|c|c|c|}
\hline \multirow{2}{*}{$\begin{array}{l}\text { Table 1 } \\
\text { Parameter }\end{array}$} & \multicolumn{3}{|c|}{ Surgical morbidity } \\
\hline & $\begin{array}{c}\text { Group I } \\
54(47.0 \%)\end{array}$ & $\frac{\text { Group II }}{61(53.0 \%)}$ & $p$ value \\
\hline Age & $61(35-89)$ & $60(44-87)$ & 0.632 \\
\hline BMI & $31.8(21.9-51)$ & $33(44.0-87)$ & 0.827 \\
\hline ASA & & & 0.562 \\
\hline 1 & $6(11.5 \%)$ & $7(11.5 \%)$ & \\
\hline 2 & $36(89.2 \%)$ & $42(68.9 \%)$ & \\
\hline 3 & $8(15.4 \%)$ & $12(19.7 \%)$ & \\
\hline 4 & $2(3.8 \%)$ & $0(0,0 \%)$ & \\
\hline Technique & & & $<0.001$ \\
\hline Laparotomy & $5(9,3 \%)$ & $0(0.0 \%)$ & \\
\hline Laparoscopy & $48(88.9 \%)$ & $45(73.8 \%)$ & \\
\hline Converted ${ }^{*}$ & $1(1.9 \%)$ & $0(0.0 \%)$ & \\
\hline Robotic & $0(0.0 \%)$ & $16(26.2 \%)$ & \\
\hline Surgical time (minutes) & $135(50.0-270.0)$ & $152(60.0-300.0)$ & 0.016 \\
\hline Blood loss (mL) & $35(0.0-500.0)$ & $20(0.0-500.0)$ & 0.386 \\
\hline Intraoperative complications & & & 1.0 \\
\hline No & $54(100 \%)$ & $60(98.4 \%)$ & \\
\hline Vascular & $0(0.0 \%)$ & $0(0.0 \%)$ & \\
\hline Urinary & $0(0.0 \%)$ & $0(0.0 \%)$ & \\
\hline Neurological & $0(0.0 \%)$ & $1(1.6 \%)$ & \\
\hline Intestinal & $0(0,0 \%)$ & $0(0.0 \%)$ & \\
\hline All & $0(0.0 \%)$ & $1(1.6 \%)$ & 1.0 \\
\hline 30-day complications & & & 0.134 \\
\hline No & $46(85.3 \%)$ & $54(88.5 \%)$ & \\
\hline 1 & $2(3.7 \%)$ & $5(8.2 \%)$ & \\
\hline II & $2(3.7 \%)$ & $0(0.0 \%)$ & \\
\hline III & $0(0.0 \%)$ & $0(0.0 \%)$ & \\
\hline IV & $t(1.9 \%)$ & $2(3.3 \%)$ & \\
\hline $\mathrm{v}$ & $3(5.6 \%)$ & $0(0.0 \%)$ & \\
\hline Yes & $8(14.8 \%)$ & $7(11.5 \%)$ & \\
\hline LLL. & & & 0.782 \\
\hline No & $54(100 \%)$ & $61(100 \%)$ & \\
\hline Yes & $0(0.0 \%)$ & $0(0.0 \%)$ & \\
\hline
\end{tabular}

\begin{tabular}{|c|c|c|c|c|c|}
\hline \multirow[t]{2}{*}{ Table 2} & \multicolumn{5}{|c|}{ Surgical morbidity } \\
\hline & No LND & LND & & Hazard ratio vs & \\
\hline Parameter & $115(46 \%)$ & $135(54 \%)$ & $p$ value & No LND $(95 \%$ CI) & $p$ value \\
\hline Age & $60(35-89)$ & $63(31-80)$ & 0.035 & & \\
\hline BMI & $32.4(21.4-51)$ & $29.85(18.0-46.3)$ & 0.003 & & \\
\hline ASA & & & 0.101 & & \\
\hline 1 & $13(11.5 \%)$ & $19(14.2 \%)$ & & & \\
\hline 2 & $78(69.0 \%)$ & $103(76.9 \%)$ & & & \\
\hline 3 & $20(17.7 \%)$ & $11(8.2 \%)$ & & & \\
\hline 4 & $2(1.8 \%)$ & $1(0.7 \%)$ & & & \\
\hline Technique & & & 0.645 & & \\
\hline Laparotomy & $5(4.3 \%)$ & $10(7.4 \%)$ & & & \\
\hline Laparoscopy & $93(80.9 \%)$ & $101(74.8 \%)$ & & & \\
\hline Converted ${ }^{*}$ & $1(0.9 \%)$ & $2(1.5 \%)$ & & & \\
\hline Robotic & $16(13.9 \%)$ & $22(16.3 \%)$ & & & \\
\hline Surgical time (minutes) & $150.0(50-300)$ & $270.0(80-600)$ & $<0.001$ & & \\
\hline Blood loss (mL) & $27.5(0-5000)$ & $80.0(0-2300)$ & $<0.001$ & & \\
\hline Intraoperative complications & & & 0.002 & & \\
\hline No & $114(99.1 \%)$ & $119(88.1 \%)$ & & & \\
\hline Vaseular & $0(0.0 \%)$ & $12(8.9 \%)$ & & & \\
\hline Urinary & $0(0.0 \%)$ & $1(0.75 \%)$ & & & \\
\hline Neurological & $1(0.9 \%)$ & $1(0.75 \%)$ & & & \\
\hline Intestinal & $0(0.0 \%)$ & $2(1.5 \%)$ & & & \\
\hline Total & $1(0.9 \%)$ & $16(11.9 \%)$ & 0.001 & $14.25(1.85-19.63)$ & 0.011 \\
\hline 30-day complications & & & 0.001 & & \\
\hline No & $100(87 \%)$ & $92(68.1 \%)$ & & & \\
\hline I & $7(6.1 \%)$ & $17(12.6 \%)$ & & & \\
\hline II & $2(1.7 \%)$ & $9(6.7 \%)$ & & & \\
\hline III & $0(0.0 \%)$ & $10(7.4 \%)$ & & & \\
\hline IV & $3(2.6 \%)$ & $3(2.2 \%)$ & & & \\
\hline $\mathrm{v}$ & $3(2.6 \%)$ & $4(3 \%)$ & & & \\
\hline Yes & $15(13 \%)$ & $43(31.9 \%)$ & $<0.001$ & $3.11(1.62-5.98)$ & 0.001 \\
\hline Major complications (III, IV or V) & $6(5.2 \%)$ & $17(12.6 \%)$ & $<0.01$ & $3.08(1.16-8.14)$ & 0.023 \\
\hline LLL. & & & $<0.01$ & $8.14(1.01-65.27)$ & 0.048 \\
\hline No & $115(100 \%)$ & $12693.3 \%)$ & & & \\
\hline Yes & $0(0.0 \%)$ & $9(6.7 \%)$ & & & \\
\hline
\end{tabular}
$0(0.0 \%)$ 